\title{
Surface kinetic energy transfer in surface quasi-geostrophic flows
}

\author{
Xavier Capet ${ }^{1,{ }^{*}}$, Patrice Klein ${ }^{2}$, Bach Lien Hua ${ }^{2}$, Guillaume Lapeyre ${ }^{3}$ and James C. \\ McWilliams $^{1}$
}

\author{
${ }^{1}$ Institute of Geophysics and Planetary Physics, UCLA, Los Angeles CA, USA \\ ${ }^{2}$ Laboratoire de Physique des Océans, IFREMER, CNRS, Plouzané, France \\ ${ }^{3}$ Laboratoire de Météorologie Dynamique, IPSL, Ecole Normale Supérieure, CNRS, Paris, France
}

*: Corresponding author : X. Capet, email address : $\underline{\text { Xavier.Capet@ifremer.fr }}$

\begin{abstract}
:
The relevance of surface quasi-geostrophic dynamics (SQG) to the upper ocean and the atmospheric tropopause has been recently demonstrated in a wide range of conditions. Within this context, the properties of SQG in terms of kinetic energy (KE) transfers at the surface are revisited and further explored. Two well-known and important properties of SQG characterize the surface dynamics: (i) the identity between surface velocity and density spectra (when appropriately scaled) and (ii) the existence of a forward cascade for surface density variance. Here we show numerically and analytically that (i) and (ii) do not imply a forward cascade of surface KE (through the advection term in the KE budget). On the contrary, advection by the geostrophic flow primarily induces an inverse cascade of surface KE on a large range of scales. This spectral flux is locally compensated by a KE source that is related to surface frontogenesis. The subsequent spectral budget resembles those exhibited by more complex systems (primitive equations or Boussinesq models) and observations, which strengthens the relevance of SQG for the description of ocean/atmosphere dynamics near vertical boundaries. The main weakness of SQG however is in the small-scale range (scales smaller than $20-30 \mathrm{~km}$ in the ocean) where it poorly represents the forward KE cascade observed in non-QG numerical simulations.
\end{abstract}

\section{Introduction}

Fundamental questions of ocean and atmosphere dynamics are how their equilibrium energy spectrum is established and what are the underlying spectral energy transfers. One difficulty is that there are a variety of contributing processes, and different fluid regions and dynamical regimes must be distinguished. In particular, regions close to boundaries, such as the ocean surface or the atmospheric tropopause, behave differently than does the interior. For boundaries Blumen (1978) developed a surface quasi-geostrophic (SQG) theory that serves as a counterpart to a model of 3-D geostrophic turbulence (Charney 1971). While the latter is driven by large-scale interior PV contrasts and is not influenced by boundary anomalies, SQG dynamics is entirely driven by the density (or potential temperature in the atmosphere) anomaly evolution at the boundary. As such, frontogenesis (in its QG limit) is the key process in SQG systems. SQG theory has been recently used to describe the 3-D dynamics of the upper troposphere (Juckes 1994; Hakim et al. 
2002; Tulloch \& Smith 2006) and the upper oceanic layers (Lapeyre \& Klein 2006; LaCasce \& Mahadevan 2006; Isern-Fontanet et al. 2006). To better understand the range of applicability of the SQG theory, Lapeyre \& Klein (2006) have further revisited theoretically and numerically the question of the coupling of the boundary dynamics (driven by the surface density) with the interior dynamics (driven by the interior PV gradients). Using the dynamical analogy (suggested by Bretherton (1966)) of the surface density as a boundary potential vorticity (PV) delta-function and using the invertibility principle of PV (Hoskins et al. 1985), Lapeyre \& Klein (2006) demonstrate the relevance of the SQG dynamics in the upper oceanic layers (the first $500 \mathrm{~m}$ ) for a spectral range extending from the smallest scales $(\mathrm{O}(10 \mathrm{~km}))$ to mesoscales (up to $\mathrm{O}(400 \mathrm{~km}))$; the larger scales are predominantly influenced by the interior PV. Such scale partition also emerges from the studies of Juckes (1994) and Tulloch \& Smith (2006) for atmospheric flows.

The spectral energy transfers at the boundaries have still to be understood. Using altimetry data Scott \& Wang (2005) computed KE fluxes that are due to nonlinear horizontal advection. They found an inverse cascade of surface kinetic energy (KE) from scales close to the Rossby deformation radius $(\approx 100-150 \mathrm{~km}$ at mid-latitudes) to larger scales. Their interpretation is that this inverse cascade mainly reflects the first baroclinic mode. In support of this hypothesis Scott \& Arbic (2007) present two-layer QG turbulent solutions for a baroclinically unstable ocean that undergoes an inverse cascade of the baroclinic (and upper layer) KE. Using PE simulations Capet et al. (2008) and Klein et al. (2008), confirm the Scott \& Wang (2005) results about the existence of a significant inverse cascade of surface KE. Their inverse cascade, again estimated from the nonlinear horizontal advection terms, extends even further down to scales around $30 \mathrm{~km}$. Yet, it is not clear that this cascade can entirely be understood in terms of QG turbulence that assumes uniform surface density. Indeed, in agreement with Lapeyre \& Klein (2006), density contrasts at the ocean surface play a leading dynamical role for scales up to at least $300 \mathrm{~km}$ (Klein et al. 2008). In this context we revisit SQG energy transfers and show that they account for an inverse surface $\mathrm{KE}$ cascade at least within the mesoscales to small scales range.

Blumen (1978) and later Held et al. (1995) noted two invariants in SQG dynamics; the surface density variance (also equal to the surface KE when appropriately dimensionalized) and the depth-integrated total energy (KE plus potential energy). By analogy with 2-D turbulence the system undergoes an inverse cascade of the total energy at low wavenumbers and a direct cascade of surface density variance at high wavenumbers. Neither Blumen (1978) nor Held et al. (1995) discussed the specific properties of the surface KE cascade in SQG theory. The focus of this paper is to characterize these properties. In Sections 2 and 3 we show (first analytically and then numerically) that despite the equivalence of surface density and velocity spectra in SQG, the details of their spectral budget differ: horizontal advection induces an upscale flux of surface KE over a large spectral range extending to small scales; whereas, it fluxes density variance downscale. The difference is compensated for by an extra term in the surface KE spectral budget that we connect to frontogenesis in Section 4. The conclusion (Section 5) examines the relevance and limitations of the SQG framework in understanding energy spectral transfers in more complex systems. 


\section{Surface KE and Density Variance Budgets}

\subsection{SQG Equations}

The SQG model ( Blumen (1978) and Held et al. (1995)) is based on a small Rossby number approximation to the PE (that describe an hydrostatic Boussinesq fluid system). All variables are nondimensionalized as in Pedlosky (1987) using $U$ and $L$ respectively the velocity and length scales, $f$ the Coriolis parameter, $N$ the Brunt-Väisälä frequency, and $H$ the depth scale. The Rossby number (defined as $\epsilon=U / f L$ ) is assumed small $(\epsilon<<1)$ and the Burger number (defined as $B=N H / f L)$ is of order one $(B=1)$. For any variable $X$, its perturbation expansion in terms of the Rossby number $\epsilon$ is $X=X^{0}+\epsilon X^{1}+\mathcal{O}\left(\epsilon^{2}\right)$. Retaining the $\mathcal{O}(1)$-terms in the momentum and hydrostatic balances leads to the resulting geostrophic and hydrostatic relations

$$
\left(u^{0}, v^{0}, \rho^{0}\right)=\left(-\Phi_{y}^{0}, \Phi_{x}^{0},-\Phi_{z}^{0}\right)
$$

where $u$ and $v$ are the horizontal velocity components respectively along the zonal $(x)$ and meridional $(y)$ coordinates. $\rho$ is the density and $z$ the vertical coordinate (with $z<0$ for an oceanic setup). The streamfunction $\Phi^{0}$ is related to pressure. SQG theory furthermore assumes that potential vorticity (PV) is uniform in the interior of the fluid and the flow decays away from the surface (i.e., $\Phi^{0} \rightarrow 0$ as $z \rightarrow-\infty$ ). The resulting PV equation in nondimensional form is

$$
\nabla_{H}^{2} \Phi^{0}+\partial_{z z} \Phi^{0}=0
$$

with the boundary condition

$$
-\left.\partial_{z} \Phi^{0}\right|_{z=0}=\rho_{s} .
$$

The subscript $s$ refers to surface variables and $\nabla_{H}$ is the horizontal gradient operator. Solving these two equations in the spectral Fourier space leads to

$$
\widehat{\Phi^{0}}(k, l, z)=-\frac{\widehat{\rho}_{s}(k, l)}{K} \exp (K z),
$$

where $\widehat{r}$ is the horizontal spectral transform. $k, l$ are the horizontal wavenumbers along the $x$ and $y$ directions and $K=\left(k^{2}+l^{2}\right)^{1 / 2}$. The dynamics at zero order and at any depth is thus entirely determined by the surface density, $\rho_{s}$. Time evolution of the flow requires one to consider the next leading order approximation (in $\epsilon$ ) to the $\mathrm{PE}$ and in particular that for surface density:

$$
\partial_{t} \rho_{s}+\boldsymbol{u}_{s}^{0} \cdot \nabla_{\boldsymbol{H}} \rho_{s}=0
$$

with $t$ the time. (2.1), (2.4) and (2.5) form a closed system, i.e., the basic SQG equation set.

Using the density equation (and retaining only the $\mathcal{O}(\epsilon)$-terms), we have at any depth

$$
w^{1}=\partial_{t} \rho^{0}+\boldsymbol{u}^{0} \cdot \nabla_{\boldsymbol{H}} \rho^{0} .
$$

Thus, from (2.4), (2.5) and (2.6), the vertical velocity in spectral space can be computed diagnostically

$$
\left.\left.\widehat{w^{1}}=-\nabla_{\boldsymbol{H}} \widehat{\cdot\left(\boldsymbol{u}_{s}^{0}\right.} \rho_{s}\right) \exp (K z)+\nabla_{\boldsymbol{H}} \widehat{\widehat{\left(\boldsymbol{u}^{0}\right.}} \rho^{0}\right) .
$$

Where $\widehat{\rho^{0}}(k, l, z)$ is given by $\widehat{\rho^{0}}(k, l, z)=\widehat{\rho}_{s}(k, l) \exp (K z)$ (using $(2.1),(2.4)$, and $\boldsymbol{\nabla}_{\boldsymbol{H}} \cdot \boldsymbol{u}^{0}=$ $0)$. Similarly, taking the z-derivative of (2.6) and using (2.1) and (2.4) provides a diagnostic expression for $w_{z}^{1}$ as a function of surface density

$$
\left.\left.\widehat{w_{z}^{1}}=-K \nabla_{\boldsymbol{H}} \widehat{\cdot\left(\boldsymbol{u}_{s}^{0}\right.} \rho_{s}\right) \exp (K z)+\nabla_{\boldsymbol{H}} \widehat{\cdot\left(\boldsymbol{u}^{0}\right.} \rho_{z}^{0}\right) .
$$


Because of the hypothesis of uniform interior, the PV horizontal and vertical structures of the flow are related to each other in this balanced model. This explains why the horizontal, as well as the vertical, velocity fields can be retrieved at all depths from only surface density(Hakim et al. 2002; Lapeyre \& Klein 2006). Another important property within the context of this study is that surface velocity and density spectra are identical. From (2.1) and (2.4), we have indeed

$$
\left|\widehat{\rho}_{s}\right|^{2}(k, l)=\left|\widehat{\mathbf{u}_{s}^{0}}\right|^{2}(k, l) .
$$

Finally, it should be noted that a difference between SQG and 2-D flows emerges from the form of the preceding equations. In SQG flows the conserved scalar is the surface density (2.5) instead of the relative vorticity, $Z=v_{x}-u_{y}$, for 2-D flows. The streamfunction at the surface is given by $\widehat{\Phi_{s}^{0}}(k, l)=-\frac{\widehat{\rho_{s}}(k, l)}{K}($ see $(2.4))$ instead of $\widehat{\Phi^{0}}(k, l)=-\frac{\widehat{Z}(k, l)}{K^{2}}$ for 2-D flows. This implies that near the surface large-scale strain in SQG plays less of a role in the advection of small-scale features, resulting in a cascade of density variance to small scales that is more local in wavenumber (Pierrehumbert et al. 1994).

\subsection{KE and Density Variance Equations}

Time evolution of the horizontal velocity, within the QG framework, is obtained by retaining only the $\mathcal{O}(\epsilon)$-terms in the momentum equations (Pedlosky 1987). At the surface the resulting equation is

$$
\partial_{t} \mathbf{u}_{s}^{0}=-\mathbf{u}_{s}^{0} \cdot \nabla \mathbf{u}_{s}^{0}-\boldsymbol{k} \wedge \mathbf{u}_{s}^{a g}
$$

where $\boldsymbol{k}$ is the unit vertical vector and $\mathbf{u}_{s}^{a g}=\mathbf{u}_{s}^{1}+\boldsymbol{k} \wedge \boldsymbol{\nabla} \Phi_{s}^{1}$ the horizontal velocity ageostrophic component. We neglect dissipation in (2.10). By spectrally manipulating (2.5) and (2.10), we get the surface kinetic energy and density variance budgets in spectral space:

$$
\begin{aligned}
& \partial_{t}\left|\widehat{\rho}_{s}\right|^{2} / 2=-\operatorname{Re}\left[\widehat{\rho}_{s}^{*}\left(\widehat{\mathbf{u}_{s}^{0} \cdot \nabla} \rho_{s}\right)\right], \\
& \partial_{t}\left|\widehat{\mathbf{u}}_{s}^{0}\right|^{2} / 2=-\operatorname{Re}\left[{\widehat{\mathbf{u}_{s}^{0}}}^{*} \cdot\left({\left.\left.\widehat{\mathbf{u}_{s}^{0} \cdot \nabla \mathbf{u}_{s}^{0}}\right)\right]-\operatorname{Re}\left[\widehat{w_{z s}^{1}} \widehat{\Phi}_{s}^{0}\right.}_{*}\right],\right.
\end{aligned}
$$

where ${ }^{*}$ is the conjugate. In $(2.12)$, we have utilized the identities

$$
{\widehat{\mathbf{u}_{s}^{0}}}^{*} \cdot \boldsymbol{k} \wedge \widehat{\mathbf{u}_{s}^{a g}}=\widehat{\mathbf{u}_{s}^{a g}} \cdot{\widehat{\nabla \Phi_{s}^{0}}}^{*}=-\widehat{\nabla \cdot \mathbf{u}_{s}^{a g}}{\widehat{\Phi_{s}^{0}}}^{*}={\widehat{w_{z s}^{1}}}_{\Phi_{s}^{0}}^{*},
$$

noting $\nabla_{H} \cdot \boldsymbol{u}_{s}^{a g}=-w_{z}^{1}$.

The nonlinear transfers due to the horizontal advection are captured by the first RHS term in (2.11) and (2.12). Integrating these equations with respect to $K$ and averaging isotropically (noted as $<.>$ ) lead us to introduce the density variance and kinetic energy spectral fluxes related to those terms, i.e.,

$$
\Pi_{\rho}=-\int_{K}^{\infty}<\operatorname{Re}\left[\widehat{\rho}_{s}^{*} \widehat{\mathbf{u}_{s}^{0} \cdot \nabla \rho_{s}}\right]>d K
$$

and

$$
\Pi_{u}=-\int_{K}^{\infty}<\operatorname{Re}\left[{\widehat{\mathbf{u}_{s}^{0}}}^{*} \cdot \widehat{\mathbf{u}_{s}^{0} \cdot \nabla \mathbf{u}_{s}^{0}}\right]>d K
$$

We also define

$$
\Pi_{a}=-\int_{K}^{\infty}<\operatorname{Re}\left[{\widehat{w_{z s}^{1}}}_{\Phi^{0}}^{*}\right]>d K,
$$

related to the last term in the RHS of (2.12), that involves ageostrophic variables. Given 

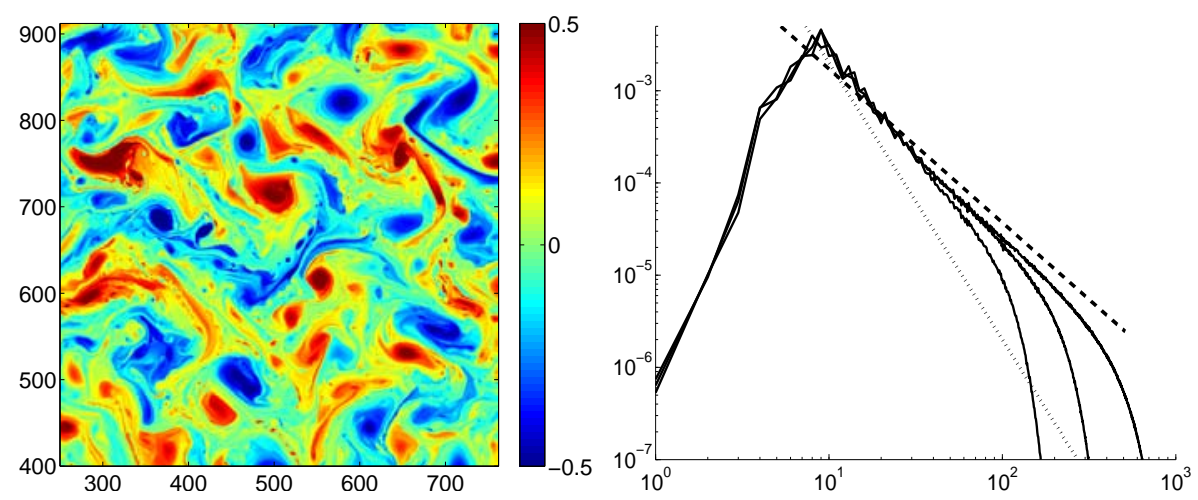

FigURE 1. (a): Snapshot of surface density at $\mathrm{t}=8$ over a $512 \times 512$ points subregion for simulation HR. (b): LR, MR, and HR spectra (continuous with high wavenumber variance increasing with resolution) of surface density variance ( i.e., also surface $\mathrm{KE}$ ) averaged over the time interval [6.4 11.2]. The dashed line (resp., dotted) represents a -5/3 (resp., -3) slope.

(2.9) the RHS of (2.11) and (2.12) are equal which yields

$$
\Pi_{\rho}=\Pi_{u}+\Pi_{a} .
$$

The relation (2.16) states that the surface density variance and KE fluxes are due to geostrophic advection (respectively $\Pi_{\rho}$ and $\Pi_{u}$ ); they differ by $\Pi_{a}$ which involves the ageostrophic flow. The striking consequence of this last term, $\Pi_{a}$, (as shown in the next section) is that the spectral transfer properties of surface $\mathrm{KE}$ and of density variance totally differ from each other despite (2.9).

\section{Numerical Simulations}

\subsection{Description}

We have performed numerical simulations for a SQG turbulent eddy field in free decay. The computation domain is doubly-periodic. The numerics are based on the model of Hua \& Haidvogel (1986) modified to solve the SQG equations set. The resolutions we employ are $512^{2}$ (LR), $1024^{2}$ (MR), and $2048^{2}$ (HR). Initial conditions are constructed by specifying a $\widehat{\phi^{0}}$ field at the surface, with random phase angles and amplitude given by $\left|\widehat{\phi_{s}^{0}}\right|^{2}(k, l)=\frac{K^{5}}{\left(K+k_{o}\right)^{12}}$. The wavenumber $k_{o}=14$ corresponds to the KE peak. Dissipation involves a biharmonic operator and a coefficient adjusted for each resolution to the minimal value for which no density variance accumulates at small scale (by inspecting the spectra).

The numerical solutions exhibit the well-known features of SQG turbulence. In the density field (Fig. 1a) small scales are energetic. This is due to frontogenesis that intensifies the density gradients and to destabilization of filaments that leads to numerous eddies having a diameter around a dozen grid points. Consequently, the density spectrum is quite flat, with a slope between $-5 / 3$ and -2 over one and half decade (Fig. 1b). Note that this description as well as the analyses performed below are for early times during which nonlinear interactions are rather strong (at a much longer time our free-decay solutions evolve toward a state where the total energy is diminished; surface spectra are steeper than -2 ; and nonlinear interactions are very weak because large isolated eddies dominate the flow). 

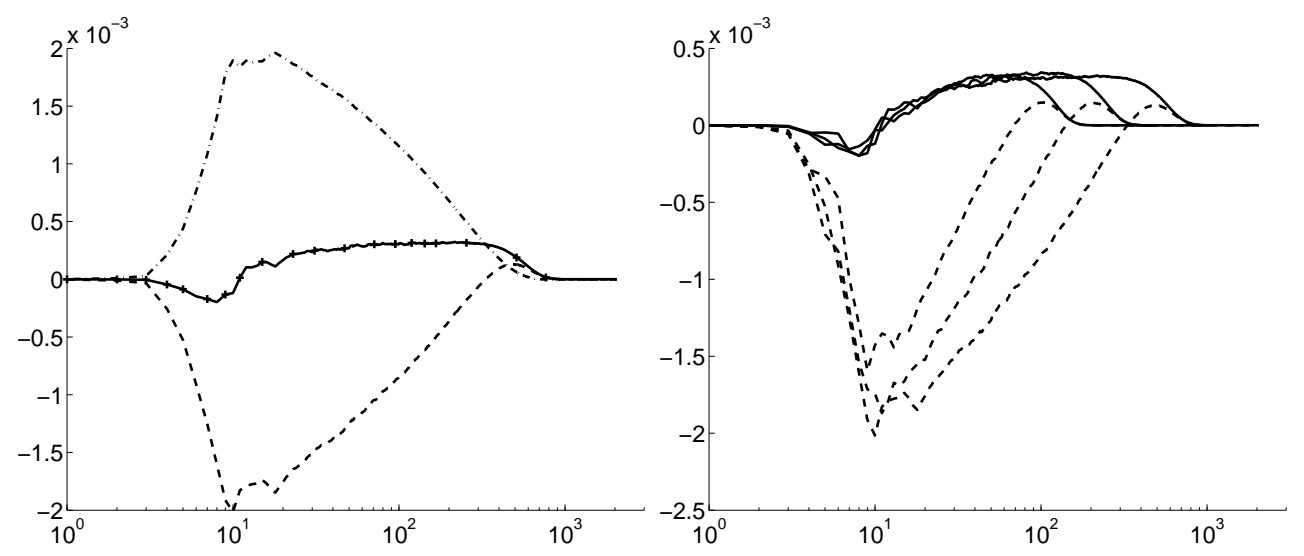

FiguRE 2. (a): HR transfer functions of surface density variance ( $\Pi_{\rho}$ : solid line) and surface KE $\left(\Pi_{u}\right.$ : dashed line and $\Pi_{a}$ : dotted-dashed line) as a function of wavenumber. Some values for the sum $\Pi_{u}+\Pi_{a}$ are also represented with '+' symbols. (b): $\Pi_{\rho}$ (solid lines) and $\Pi_{u}$ (dashed lines) for the three different resolutions (LR, MR, and HR).

\subsection{Spectral Transfers of Surface KE and Density Variance}

The different terms (2.13), (2.14) and (2.15) are computed from 10 snapshots of density (taken over six eddy turnover time units during which an inertial range is most cleanly present in the density spectral transfers). $w_{z}^{1}$ (required to compute $\Pi_{a}$ ) is diagnosed from (2.8). Results are given for HR in Fig. 2a. The forward cascade of density variance extends roughly from $k=15$ to the dissipation scale $(k>500)$. This is the classical result anticipated by Blumen (1978) and subsequently verified by numerous studies (Celani et al. (2004)). The flux is close to constant over this interval. The advective flux of surface $\mathrm{KE}$, on the other hand, is upscale $\left(\Pi_{u}<0\right)$ over a large spectral range. To our knowledge this important aspect of SQG turbulence had been overlooked. A downscale flux is also present but it concerns a narrow range of very small scales (with wavenumbers larger than $k=300)$. From (2.16) the difference between $\Pi_{\rho}$ and $\Pi_{u}$ is entirely explained by $\Pi_{a}$ (whose physical interpretation is discussed in the next section). This has been checked by plotting some values of $\Pi_{u}+\Pi_{a}$ in Fig. 2a. Unlike $\Pi_{\rho}, \Pi_{u}$ and $\Pi_{a}$ have no plateau.

In Fig. $2 \mathrm{~b}, \Pi_{u}$ and $\Pi_{\rho}$ are plotted for different horizontal resolutions. For $\Pi_{\rho}$, increasing the resolution simply extends its plateau corresponding to a forward cascade of density variance over a wider spectral range. The same behavior is observed for the the upscale surface KE advective flux, which indicates its robustness. On the other hand, the downscale surface KE flux is not robust with respect to the resolution changes. As resolution increases, the region where $\Pi_{u}$ is positive moves to higher wavenumber and roughly follows the dissipation range. In other words, the downscale advective flux of surface $\mathrm{KE}$ would be absent from a solution with infinite resolution.

\section{Discussion}

Although surface density and velocity spectra are identical over a large spectral range (as confirmed by their tendency terms), their dynamics revealed by their budgets are quite different because of the term $\Pi_{a}$. The physical meaning of $\Pi_{a}$ can be understood in terms of surface frontogenesis. Let us consider a front undergoing frontogenesis as

represented in Fig. 3. In the following reasoning we assume that $\Phi^{0}$ has a zero horizontal 


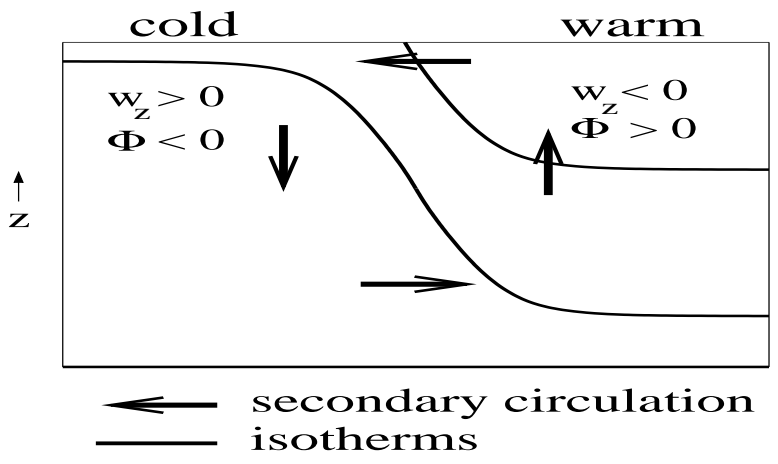

FigURE 3. Schematic of the frontogenetic situation described in Sec 4. This situation represents an intensifying density front embedded in a horizontal deformation field near the ocean surface (top of the figure) with light/warm water on the right-hand side. The underlying ageostrophic secondary circulation (arrows) counteracts frontal intensification (Hoskins \& Bretherton 1972) with $\Phi w_{z}<0$ on either side of the front near the surface.

average at the surface because $\Phi^{0}$ is a streamfunction anomaly. Since $\partial_{z} \Phi^{0}=-\rho^{0}$ and velocities are vanishing at depth, we shall have $\Phi^{0}>0$ (resp., $\left.\Phi^{0}<0\right)$ at the surface on the warm (resp., cold) side. As for the ageostrophic secondary circulation, it is made of an ascending branch on the warm side and a descending branch on the cold side (Hoskins $\&$ Bretherton 1972). The condition $w=0$ at the surface yields $\Phi^{0} w_{z}^{1}<0$ on both sides of the front. Since frontogenesis statistically dominates over frontolysis in the wavenumber range, where the forward cascade of surface density variance is taking place, we expect $-\widehat{w_{z}^{1}} \cdot \widehat{\Phi}^{0}{ }^{*}$ to be positive there; this is consistent with the shape of $\Pi_{a}$ (Fig. 2a).

A more quantitative analysis allows us to relate $\Pi_{a}$ to the release of available potential energy by the frontogenesis mechanisms. This is done by using (2.6), (2.10) (valid at any depth), and the thermal wind balance (deduced from the geostrophic and hydrostatic approximations (2.1)):

$$
\nabla_{\boldsymbol{H}} \rho=\boldsymbol{k} \wedge \partial_{z} \boldsymbol{u}^{0}
$$

Taking $\boldsymbol{\nabla}_{\boldsymbol{H}}$ of (2.6) leads to

$$
\frac{d \boldsymbol{\nabla}_{\boldsymbol{H}} \rho}{d t}=-\boldsymbol{Q}+\boldsymbol{\nabla}_{\boldsymbol{H}} w^{1} .
$$

with $\boldsymbol{Q}=\left[\boldsymbol{\nabla}_{\boldsymbol{H}} \boldsymbol{u}^{0}\right]^{T} \cdot \boldsymbol{\nabla}_{\boldsymbol{H}} \rho^{0}$ ([ $]^{T}$ is the transpose matrix) the frontogenetic vector. Subtracting $\boldsymbol{k} \wedge \partial_{z}$ of (2.10) from (4.2), and using the thermal wind balance yields (Hoskins \& Bretherton 1972; Klein et al. 1998)

$$
2 \boldsymbol{Q}=\nabla_{H} w^{1}-\mathbf{u}_{z}^{a g} .
$$

(4.3), that comprises the QG version of the Eliassen-Sawyer equation (Thomas \& Lee 2005), leads to

$$
\frac{2}{K} \cdot \widehat{\boldsymbol{Q}} \cdot{\widehat{\nabla} \boldsymbol{H} \rho^{0}}^{*}=K{\widehat{w^{1}}}_{\rho^{0}}^{*}-\frac{1}{K} \widehat{\boldsymbol{u}_{z}^{a g}} \cdot{\widehat{\nabla} \boldsymbol{H} \rho^{0}}^{*} .
$$

However, (4.4) can also be written as (using $\nabla_{H} \cdot \boldsymbol{u}^{a g}=-w_{z}^{1}$ )

$$
\frac{2}{K} \cdot \widehat{\boldsymbol{Q}} \cdot{\widehat{\nabla} \boldsymbol{H} \rho^{0}}^{*}=\left[\widehat{w_{z}^{1}}{\widehat{\Phi^{0}}}^{*}-\widehat{w^{1}} \widehat{\Phi}_{z}^{*}\right]_{z} .
$$

Integrating (4.5) over the whole water column (with $w^{1}=0$ at the boundaries and $\Phi^{0}=0$ 
at the bottom) and over $K$, yields

$$
-\int_{K}^{\infty}<\operatorname{Re}\left[\frac{2}{K} \int_{-\infty}^{0} \widehat{\boldsymbol{Q}} \cdot{\widehat{\nabla_{\boldsymbol{H}} \rho^{0}}}^{*} d z\right]>d K=-\int_{K}^{\infty}<\operatorname{Re}\left[\left.{\widehat{w^{1}}}_{z}{\widehat{\Phi^{0}}}^{*}\right|_{z=0}\right]>d K=\Pi_{a},
$$

which was verified in our numerical solutions. The same integration but involving (4.4) yields

$$
\Pi_{a}=\int_{K}^{\infty}<\operatorname{Re}\left[-K \int_{-\infty}^{0}{\widehat{w^{1}}}_{\rho^{0}}^{*} d z+\frac{1}{K} \int_{-\infty}^{0}{\widehat{\boldsymbol{u}_{z}^{g}}}_{\nabla_{\boldsymbol{H}} \rho^{0}}^{*} d z\right]>d K .
$$

(4.6) indicates that $\Pi_{a}$ is directly related to the production of density gradients within the fluid. This term should be positive in particular in the high wavenumber region, where density gradients are the strongest (since density gradient spectrum has a $k^{1 / 3}$ slope). (4.6) bears some similarity with a relation found by Lapeyre et al. (2006) that couples frontogenesis and restratification (through $w_{z} \rho$ ) and redistributes density vertically. (4.7) further indicates that $\Pi_{a}$ is related to the release of available potential energy by the ageostrophic circulation associated with frontogenesis. Indeed, the first term on the right hand side of (4.7) corresponds to a buoyancy flux while the second term reflects the manner ageostrophic flows modify the stratification through differential horizontal advection. For frontogenetic conditions (Fig. 3), both of these terms are positive and lead to a reduction of the available potential energy. Thus $\Pi_{a}$, directly related to the production of density gradients within the fluid, can be interpreted as a transformation of available potential energy into surface KE. ¿From (2.16) the consequence of the positiveness of both $\Pi_{a}$ (related to the frontogenesis strength) and $\Pi_{\rho}$ (related to the direct cascade of density variance) and of the dominance of $\Pi_{a}$ is that $\Pi_{u}$ (related to the nonlinear advective transfers of surface $\mathrm{KE}$ ) must be negative over most of the spectral range. These findings emphasize the importance of small-scale frontogenesis in the surface KE budget (also noted in Capet et al. (2008) and Klein et al. (2008)).

\section{Conclusion}

We have revisited the spectral transfer properties of the SQG theory. Although the velocity and density spectra at the surface are identical, their spectral budgets differ. Surface density variance experiences a forward cascade as stated by Blumen (1978) and confirmed by numerous subsequent studies. Surface KE on the other hand experiences a clear and significant inverse cascade on a large range of scales, i.e., a transfer through advection by the geostrophic flow from small to larger scales. This apparent contradiction is explained by the presence in the surface KE budget of an additional term associated with the ageostrophic component of the flow that acts as a source term. Its main underlying physics is the restoration of the thermal wind balance, tending to be destroyed by the frontogenesis processes near the surface.

In systems more complex than SQG, surface density and velocity spectra have also been found to remarkably coincide, at least over a large part of the submesoscale and mesoscale range as shown in the PE framework by Klein et al. (2008) (see Fig. 4a that reproduces their spectra; their simulations are for a baroclinically unstable quasi-equilibrated jet in a periodic channel). But again the similarity of these surface spectra does not imply a similarity of their respective advective fluxes. In fact, and as found in SQG, surface density variance is fluxed downscale, whereas $\mathrm{KE}$ is fluxed upscale over a wide central part of the wavenumber range (Fig.4b). The KE advective flux is compensated for by an ageostrophic term whose expression is similar to $\Pi_{a}$. The main difference with our 

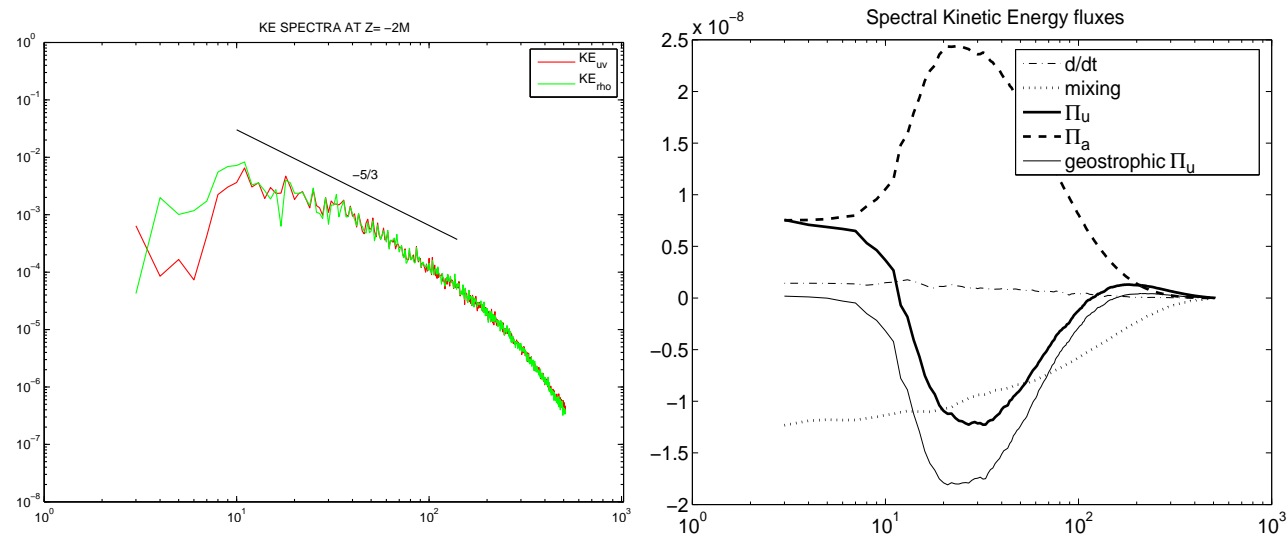

FiguRE 4. (a): Surface velocity spectrum (red curve) and density spectrum normalized by $\left[g /\left(N \rho_{0}\right)\right]^{2}$ (green curve) estimated from a high-resolution PE simulation (from Klein et al. $(2008)) . k=10$ corresponds to a wavelength of $300 \mathrm{~km}$. (b): Surface kinetic energy transfer functions (from Klein et al. (2008)): $\Pi_{u}$ calculated directly from the total (divergent plus non-divergent components) velocities $u$ and $v$ (thick solid curve); $\Pi_{u}$ calculated from the non-divergent (geostrophic) velocities (thin solid curve); $\Pi_{a}$ (dashed curve). The dashed-dot curve corresponds to the tendency and the dotted curve to the mixing terms contribution.

SQG results is that nonlinear interactions involve advection of geostrophic quantities by both the geostrophic and ageostrophic velocities. As a result, both $\Pi_{a}$ and $\Pi_{u}$ give a net contribution to the surface KE budget for PE solutions since they are non-zero for the lowest wavenumbers. These results corroborate previous evidence of a strong impact of the ageostrophic circulation on near-surface KE spectral transfers (Capet et al. 2008; Klein et al. 2008).

Overall the comparison between SQG and PE energy transfers suggest SQG as a pertinent framework to understand the inverse KE cascade found by Scott \& Wang (2005) using altimetry data. A rapidly growing body of numerical (Lapeyre \& Klein 2006; Klein et al. 2008) and observational (LaCasce \& Mahadevan 2006; Isern-Fontanet et al. 2006; Le Traon et al. 2007) evidence indicates that the ocean sea level often does significantly reflect surface modes (as opposed to primarily the interior first baroclinic and barotropic modes Scott \& Arbic 2007) even for scales above the deformation radius.

One weakness of SQG is that it exhibits a downscale surface KE flux for high wavenumbers that is weak and resolution-dependent (it is basically confined to the dissipation range). On the other hand, high resolution PE (Capet et al. 2008) or Boussinesq (Molemaker et al. 2007) solutions produce robust downscale surface KE within the small-scale range $(<20-30 \mathrm{~km})$. This discrepancy can be traced to momentum advection by the ageostrophic flow as shown in Fig.4b, where it can be seen that the advective KE flux associated with the total flow is always greater than that associated with its geostrophic component (see also Capet et al. 2008). Molemaker et al. (2007) further relate the nonQG effects to ageostrophic frontal instabilities at submesoscale. This downscale flux of $\mathrm{KE}$ at submesoscale inadequately reproduced within the QG approximations may play an essential role in the dissipation of oceanic energy.

This work is supported by the CNRS and IFREMER (FRANCE) and by ONR (USA). PK and BLH also acknowledge the support from the French ANR (Agence Nationale pour la Recherche, Contract no ANR-05-CIGC-010). We thank two anonymous reviewers for their thoughful comments on the manuscript. 


\section{REFERENCES}

Blumen, W. 1978 Uniform potential vorticity flow: Part I. Theory of wave interactions and two-dimensional turbulence. J. Atmos. Sci. 35, 774-783.

Bretherton, F.P. 1966 Critical layer instability in baroclinic flows. Quart. J. Roy. Meteor. Soc. 92, 325-334.

Capet, X., McWilliams, J.C., Molemaker, M.J. \& Shchepetkin, A. 2008 Mesoscale to submesoscale transition in the California Current System: Energy balance and flux. J. Phys. Ocean. ., Submitted.

Celani, A., Cencini, M., Mazzino, A. \& Vergassola, M. 2004 Active and passive tracers face to face. New J. Phys. 6, 72 .

Charney, J.G. 1971 Geostrophic turbulence. J. Atmos. Sci. 28, 1087-1095.

Hakim, G., Snyder, C. \& Muraki, D. 2002 A new surface model for cyclone-anticyclone asymmetry. J. Atmos. Sci. 59, 2405-2420.

Held, I., Pierrehumbert, R., Garner, S. \& Swanson, K. 1995 Surface quasi-geostrophic dynamics. J. Fluid Mech. 282, 1-20.

Hoskins, B. \& Bretherton, F. 1972 Atmospheric frontogenesis models: Mathematical formulation and solution. J. Atmos. Sci. 29, 11-37.

Hoskins, B., McIntyre, M. \& Robertson, A. 1985 One the use and significance of isentropic potential vorticity maps. Quart. J. Roy. Meteor. Soc. 111, 877-946.

HuA, B.L. \& HAIDVOGEL, D.B. 1986 Numerical simulations of the vertical structure of quasigeostrophic turbulence. J. Atmos. Sci. 43, 2923-2936.

Isern-Fontanet, J., Chapron, B., Lapeyre, G. \& Klein, P. 2006 Potential use of microwave sea surface temperature for the estimation of oceanic currents. Geophys. Res. Lett. 12, 33(24), 11-15.

Juckes, M. 1994 Quasigeostrophic dynamics of the tropopause. J. Atmos. Sci. 51, 2756-2768.

Klein, P., Hua, B.L., Lapeyre, G., Capet, X., Gentil, S. Le \& Sasaki, H. 2008 Upper ocean turbulence form high 3-d resolution simulations. J. Phys. Ocean. ., in press.

Klein, P., TrÉGuier, A-M. \& HuA, B.L. 1998 Three-dimensional stirring of thermohaline fronts. J. Mar. Res. 56, 589-612.

LaCAsce, J.H. \& Mahadevan, A. 2006 Estimating subsurface horizontal and vertical velocities from sea surface temperature. J. Mar. Res. 27, 695-721.

Lapeyre, G. \& Klein, P. 2006 Dynamics of the upper oceanic layers in terms of surface quasigeostrophy theory. J. Phys. Ocean. 36, 165-176.

Lapeyre, G., Klein, P. \& HuA, B.L. 2006 Oceanic restratification forced by surface frontogenesis. J. Phys. Ocean. 36, 1577-1590.

Le Traon, P.Y., Klein, P., Hua, B.L. \& Dibarboure, G. 2007 Do altimeter data agree with interior or surface quasi-geostrophic theory. J. Phys. Ocean. p. In press.

Molemaker, M.J., McWilliams, J.C. \& CAPet, X. 2007 Balanced and unbalanced routes to dissipation in an equilibrated eady flow. J. Fluid Mech. ., Submitted.

Pedlosky, J. 1987 Geophysical Fluid Dynamics. Springer-Verlag.

Pierrehumbert, R.T., Held, I.M. \& Swanson, K.L. 1994 Spectra of local and nonlocal two-dimensional turbulence. Chaos, Solitons and Fractals 4, 1111-1116.

ScotT, R.B. \& ARBIC, B.K. 2007 Spectral energy fluxes in geostrophic turbulence: implications for ocean energetics. J. Phys. Ocean. 37, 673-688.

Scott, R. \& WAng, F. 2005 Direct evidence of an oceanic inverse kinetic energy cascade from satellite altimetry. J. Phys. Ocean. 35, 1650-1666.

Thomas, L. \& LeE, C. 2005 Intensification of ocean fronts by down-front winds. J. Phys. Ocean. 35, 1086-1102.

Tulloch, R. \& Smith, K. S. 2006 A new theory for the atmospheric energy spectrum: Depthlimited temperature anomalies at the tropopause. Proc. Natl. Acad. Sci. USA 103, 14690-14694. 